\title{
Community-acquired Pseudomonas aeruginosa sacro-iliitis in a previously healthy patient
}

\author{
L. CALZA, R. MANFREDI, G. MARINACCI, L. FORTUNATO and F. CHIODO \\ Department of Clinical and Experimental Medicine, Division of Infectious Diseases, University of Bologna, S. \\ Orsola Hospital, I-40138 Bologna, Italy
}

\begin{abstract}
Pyogenic sacro-iliitis is an uncommon osteo-articular infection that occurs usually in immunocompromised patients and is associated with gram-positive cocci. It is very rarely linked with a gram-negative aetiology. The first case of Pseudomonas aeruginosa sacro-iliitis is described, which occurred in a previously healthy young man, without history of prior traumatic events, hospitalisation or chronic underlying disease.
\end{abstract}

\section{Introduction}

The incidence of bacterial haematogenous osteomyelitis has increased in recent years, probably as a consequence of a rise in the frequency of pyogenic non-tuberculous bacterial infections due to invasive procedures, hospitalisation and prolonged survival of immunocompromised patients [1].

Haematogenous osteomyelitis is caused by a single organism in $95 \%$ of cases, and Staphylococcus aureus is the most frequent agent responsible for nontuberculous bacterial infections of bone. However, recent reports emphasise the emerging role of gramnegative bacilli, including Pseudomonas aeruginosa, which is the most frequently isolated gram-negative organism in chronic osteomyelitis [1,2]. Osteo-articular infectious complications due to $P$. aeruginosa are usually difficult to treat, because they generally involve immunocompromised or hospitalised patients at serious risk. There is also a high tendency for these organisms to develop multiple antibiotic resistance $[2,3]$.

Several cases of pelvic and vertebral osteomyelitis associated with $P$. aeruginosa have been reported, but here we describe, to the best of our knowledge, the first case of sacro-iliitis due to this bacterium.

\section{Case report}

A previously healthy 33-year-old male was admitted to

Received 30 Oct. 2001; revised version received 11 Feb. 2002; accepted 15 Feb. 2002.

Corresponding author: Dr L. Calza (e-mail: malinf@med. unibo.it). hospital with a 10-day history of intermittent fever with chills, malaise, fatigue, lack of appetite and persistent pain in the left hip and around the sacral region. He did not use drugs, never drank alcohol or smoked, and did not have any prior local trauma, surgery or hospitalisation. On admission to the hospital, his body temperature was $38.2^{\circ} \mathrm{C}$, the liver was enlarged but the spleen was not palpable. Cardiac and pulmonary auscultation did not disclose any abnormality. Physical examination revealed a marked tenderness of the left coxo-femoral articulation, and both active and passive movement of the left leg proved very painful. The laboratory tests showed: white blood cell (WBC) count, 9250/ $\mu$ l, with $82 \%$ segmented neutrophils; CD4+ lymphocyte count, $612 / \mu 1$; haemoglobin, $11.1 \mathrm{~g} / \mathrm{dl}$; haematocrit, $37 \%$; erythrocyte sedimentation rate (ESR), $54 \mathrm{~mm} / \mathrm{h}$. Urinalysis, electrocardiogram, echocardiographic study and chest X-ray did not reveal any significant abnormalities; abdominal ultrasonography showed a slight hepatomegaly. Blood and urine cultures were repeatedly negative. Anti-HIV antibody test was negative. Pelvic X-ray examination was normal but pelvic magnetic resonance imaging (MRI) revealed an increased joint space with moderate effusion and diffuse bony irregularities at the left sacro-iliac articulation (Fig. 1). An ${ }^{111}$ indium-labelled WBC scan disclosed focal uptake in the same area, confirming the diagnosis of sacro-iliitis. CT-guided percutaneous drainage of the articular effusion and bone lesion was performed and a purulent exudate was discovered. Culture of this exudate yielded a pure growth of $P$. aeruginosa which was susceptible to piperacillin-tazobactam, ticarcillinclavulanate, cefotaxime, ceftazidime, imipenem, aztreonam, aminoglycosides and fluoroquinolones, but resistant to ampicillin, amoxicillin, mezlocillin, firstand second-generation cephalosporins, tetracyclines and co-trimoxazole. 


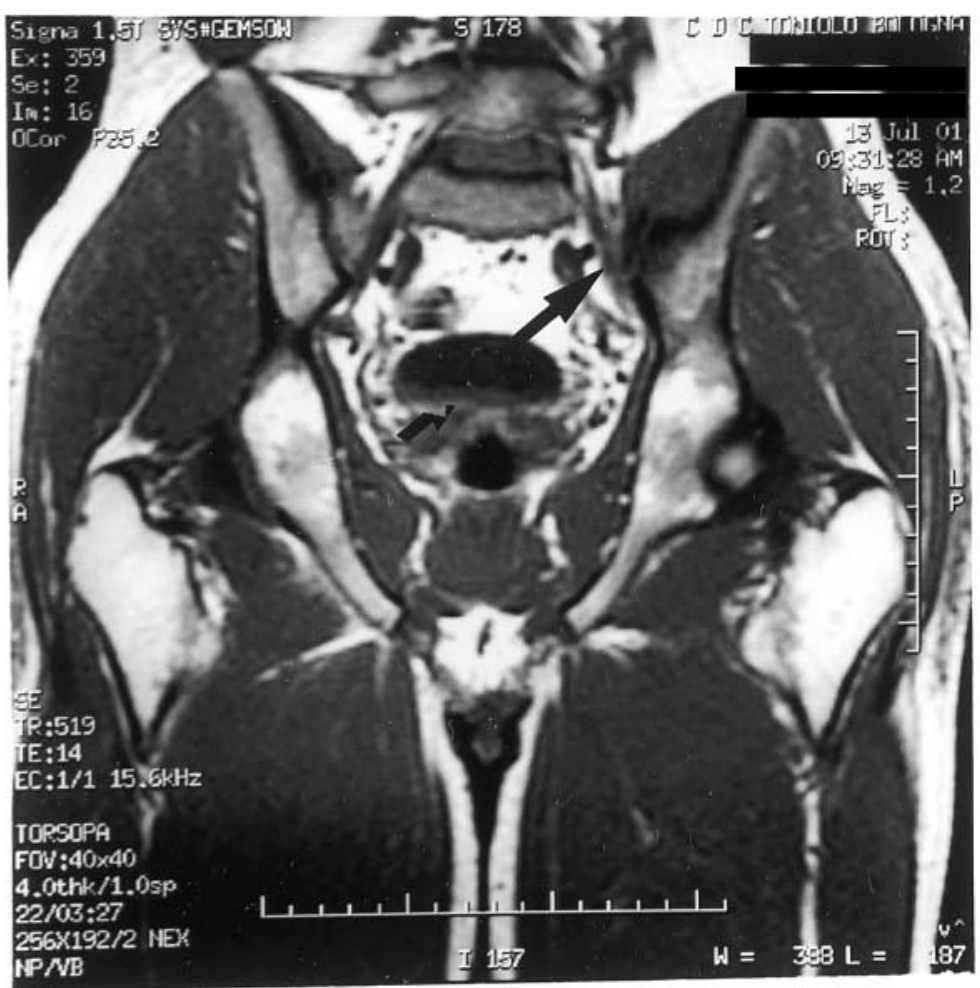

Fig. 1. Pelvic MRI showing moderate effusion and diffuse bony irregularities (arrow) at the left sacro-iliac joint, consistent with sacro-iliitis.

The patient was treated with oral ciprofloxacin $(500 \mathrm{mg}$ twice a day) and intravenous imipenem $(1 \mathrm{~g}$ three times daily) for 6 weeks. He became afebrile with disappearance of systemic symptoms and pain in the left hip within 12 days. ESR and other laboratory findings returned to normal levels in 3 weeks. Nevertheless, a repeated pelvic MRI 6 weeks after the start of antimicrobial therapy disclosed the persistence of inflammatory signs at the left sacro-iliac joint, in association with diffuse irregularities of the articular bone margins. Therefore, antibiotic treatment was modified. Ciprofloxacin and imipenem were replaced with intravenous piperacillin-tazobactam $(4.5 \mathrm{~g}$ twice daily) and amikacin (450 mg twice daily) and administered for a further 6 weeks. The last pelvic MRI, which was performed 12 weeks after the first MRI, showed complete resolution of the articular effusion and concurrent bone lesions.

\section{Discussion}

$P$. aeruginosa is an aerobic, motile, non-fermentative gram-negative bacillus, widespread in the environment and usually regarded as a trivial commensal of the skin, mucous membranes and intestinal tract. However, it is an opportunist pathogen, responsible for pyogenic infections in immunocompromised or hospitalised persons [1, 2, 4]. Osteo-articular haematogenous infectious complications caused by $P$. aeruginosa generally include pelvic and vertebral, acute and chronic osteomyelitis. These occur in patients with primary or secondary immunodeficiency, underlying chronic diseases, prior prolonged broad-spectrum antibiotic therapy, intravenous drug abuse, or those undergoing genitourinary or other invasive procedures $[1,3]$. $P$. aeruginosa may also lead to post-traumatic or postoperative osteo-articular infections, resulting from direct inoculation of bone with the organisms, which may progress to chronic osteomyelitis [2].

Pyogenic sacro-iliitis is a very rare osteo-articular infection, generally occurring in immunocompromised patients, and caused by gram-positive cocci, such as $S$. aureus and Streptococcus pneumoniae. Sacro-iliac infection caused by gram-negative pathogens is very rare and to the best of our knowledge no cases of $P$. aeruginosa sacro-iliitis have been reported to date [5]. The common clinical presentation of sacro-iliac pyogenic infection includes fever and other systemic symptoms (chills, malaise, asthenia, anorexia and weight loss), in association with sacral, hip or leg pain and limitation of motion, inability to bear weight and local inflammation. All patients have an increased ESR but leukocytosis is variable $[5,6]$. Pelvic plain radio-

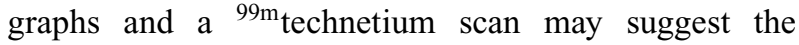
diagnosis, but they can also fail to show pathological changes in both bone tissue and the sacro-iliac joint, probably because of the low sensitivity of these techniques during the first weeks of the disease. On the other hand, contrast-enhanced CT scan, MRI and an 111 indium-labelled WBC scan represent the best 
diagnostic imaging assays, which allow early detection of bone and articular abnormalities occurring during the initial phases of osteomyelitis [5,7-9]. Culture of blood and percutaneous drainage of osteo-articular lesions may lead to bacterial isolation and microbiological diagnosis [9].

Currently, the antimicrobial treatment of $P$ aeruginosa bone infections still represents a major therapeutic challenge because of several factors. These include the poor bone penetration of many antibiotics and the presence of abscesses, sequestra and foreign bodies. In addition, bacterial glycocalyx and slime production lead to the formation of a micro-environment that is impervious to antibiotics. Furthermore, many strains show a high tendency to develop multiple antibiotic resistance. Some parenteral antibiotics have been used as monotherapy to treat $P$. aeruginosa osteomyelitis, including ticarcillin-clavulanate, imipenem and ceftazidime. Oral or parenteral fluoroquinolones have also been used successfully $[2,9,10]$.

Nevertheless, there is a high risk of development of bacterial resistance when fluoroquinolones or other antimicrobial compounds are administered as single therapeutic agents, while short-term regimens may result in clinical relapse. The long-term administration of dual parenteral antibiotics is now considered the most effective antimicrobial therapy for preventing relapses and the emergence of resistant bacteria. Specifically, the combination of a $\beta$-lactam compound with an aminoglycoside or a fluoroquinolone, administered for 6-8 weeks, is widely considered the most appropriate treatment for $P$. aeruginosa osteomyelitis $[2,10]$. Surgical debridement in addition to antibiotic therapy may be necessary for extensive, persisting lesions characterised by bone sequestra, as it can favour the action of antimicrobial compounds [7].
In conclusion, to our knowledge this patient represents the first described case of sacro-iliitis due to $P$. aeruginosa and, unlike previously reported cases of pyogenic sacro-iliitis, it occurred in an immunocompetent and previously healthy young man. This osteoarticular infection, although remaining a rare event, should be carefully considered in selected situations characterised by increased risk, due to its diagnostic and especially therapeutic challenge.

\section{References}

1. Chevalier X, Lavabre C, Claudepierre P, Larget-Piet B. Iatrogenically induced vertebral osteomyelitis due to Pseudomonas aeruginosa. Clin Exp Rheumatol 1996; 14: 191-194.

2. Lucht RF, Fresard A, Berthelot $\mathrm{P}$ et al. Prolonged treatment of chronic Pseudomonas aeruginosa osteomyelitis with a combination of two effective antibiotics. Infection 1994; 22: 276-280.

3. Hancock REW. Resistance mechanisms in Pseudomonas aeruginosa and other nonfermentative gram-negative bacteria. Clin Infect Dis 1998; 27 Suppl 1: S93-S99.

4. Mader R, Yeromenco E. Pseudomonas osteomyelitis of the symphysis pubis after inguinal hernia repair. Clin Rheumatol 1999; 18: 167-169.

5. Babinchak TJ, Riley DK, Rotheram EB. Pyogenic vertebral osteomyelitis of the posterior elements. Clin Infect Dis 1997; 25: $221-224$.

6. Hedstrom SA, Lidgren L. Acute hematogenous pelvic osteomyelitis in athletes. Am J Sports Med 1982; 10: 44-46.

7. De Miguel J, Collazos J, Ayarza R, Abaitua JM, Quilez J. Pubic osteomyelitis due to Pseudomonas aeruginosa. Clin Infect Dis 1993; 17: 515-516.

8. Lau LS, Bin G, Jaovisidua S, Dankner W, Sartoris DJ. Cost effectiveness of magnetic resonance imaging in diagnosis of Pseudomonas aeruginosa infection after puncture wound. J Foot Ankle Surg 1997; 36: 36-43.

9. Carek PJ, Dickerson LM, Sack JL. Diagnosis and management of osteomyelitis. Am Fam Physician 2001; 63: 2413-2420.

10. Galanakis N, Giamarellou H, Moussas T, Dounis E. Chronic osteomyelitis caused by multi-resistant gram-negative bacteria: evaluation of treatment with newer quinolones after prolonged follow-up. J Antimicrob Chemother 1997; 39: 241-246. 